\title{
Expanding the Chemistry of Salt-Inclusion Materials: Utilizing the Titanyl Ion as a Structure Directing Agent for the Targeted Synthesis of Salt-Inclusion Titanium Silicates
}

\author{
Gregory Morrison, Hans-Conrad zur Loye* \\ Center for Hierarchical Waste form Materials, Columbia, South Carolina 29208 \\ Department of Chemistry and Biochemistry, University of South Carolina, Columbia, SC, 29208 \\ *Corresponding author. E-mail: zurLoye@mailbox.sc.edu
}


Table S1. Atomic \% of each element present in the reported crystals based on EDS. Note that these results should not be viewed as quantitative. In particular, the F peak overlaps with a Cs peak and has low intensity, often making it not-detectable (nd).

\begin{tabular}{|c|c|c|c|c|c|c|c|c|}
\hline \multirow[b]{2}{*}{ Compound } & \multirow[b]{2}{*}{ K } & \multicolumn{7}{|c|}{ Atomic Percent $(\%)$} \\
\hline & & $\mathrm{Rb}$ & $\mathrm{Cs}$ & $\mathrm{Ti}$ & $\mathrm{Si}$ & $\mathrm{O}$ & $\mathrm{F}$ & $\mathrm{Cl}$ \\
\hline (1) & $\mathrm{n} / \mathrm{a}$ & n.a & 16.35 & 7.64 & 21.41 & 52.76 & nd & 1.84 \\
\hline (2) & $\mathrm{n} / \mathrm{a}$ & $\mathrm{n} / \mathrm{a}$ & 18.66 & 14.64 & 15.12 & 49.42 & nd & 2.16 \\
\hline (3) & $\mathrm{n} / \mathrm{a}$ & 10.05 & $\mathrm{n} / \mathrm{a}$ & 7.13 & 20.79 & 59.98 & 0.14 & 1.91 \\
\hline Cs9-SIM_KCl & 15.04 & $\mathrm{n} / \mathrm{a}$ & 0 & 9.27 & 15.39 & 59.61 & nd & 0.68 \\
\hline Cs9-SIM_KNO 3 & 10.97 & $\mathrm{n} / \mathrm{a}$ & 0.71 & 5.85 & 17.08 & 64.02 & nd & 1.36 \\
\hline $\mathrm{Rb}-\mathrm{SIM} \mathrm{KNO}_{3}$ & 13.26 & 0 & $\mathrm{n} / \mathrm{a}$ & 7.74 & 18.64 & 58.57 & nd & 1.80 \\
\hline
\end{tabular}


Table S2. Interatomic distances and bond valence sums for the titanium, silicon, and halide (X) polyhedra in $\left[\mathrm{Cs}_{3} \mathrm{Cs}_{6} \mathrm{Cl}_{0.7} \mathrm{~F}_{0.3}\right]\left[(\mathrm{TiO})_{4}\left(\mathrm{Si}_{12} \mathrm{O}_{32}\right)\right]$ (1).

\begin{tabular}{|c|c|c|}
\hline Polyhedron & Interaction & Distance \\
\hline \multirow[t]{5}{*}{$\mathrm{Ti}(1) \mathrm{O}_{6}$} & $\mathrm{Ti}(1)-\mathrm{O}(1)$ & $1.670(3)$ \\
\hline & $\mathrm{Ti}(1)-\mathrm{O}(3)$ & $1.957(3)$ \\
\hline & $\mathrm{Ti}(1)-\mathrm{O}(2)$ & $1.968(3)$ \\
\hline & $\mathrm{Ti}(1)-\mathrm{O}(8)$ & $1.979(3)$ \\
\hline & $\mathrm{Ti}(1)-\mathrm{O}(7)$ & $1.983(3)$ \\
\hline \multirow[t]{4}{*}{$\mathrm{Si}(1) \mathrm{O}_{4}$} & $\mathrm{Si}(1)-\mathrm{O}(2)$ & $1.579(3)$ \\
\hline & $\mathrm{Si}(1)-\mathrm{O}(4)$ & $1.5949(9)$ \\
\hline & $\mathrm{Si}(1)-\mathrm{O}(6)$ & $1.6213(15)$ \\
\hline & $\mathrm{Si}(1)-\mathrm{O}(5)$ & $1.626(3)$ \\
\hline \multirow{4}{*}{$\overline{\mathrm{Si}(2) \mathrm{O}_{4}}$} & $\mathrm{Si}(2)-\mathrm{O}(3)$ & $1.584(3)$ \\
\hline & $\mathrm{Si}(2)-\mathrm{O}(7)$ & $1.594(3)$ \\
\hline & $\mathrm{Si}(2)-\mathrm{O}(5)$ & $1.648(3)$ \\
\hline & $\mathrm{Si}(2)-\mathrm{O}(9)$ & $1.649(3)$ \\
\hline \multirow[t]{4}{*}{$\mathrm{Si}(3) \mathrm{O}_{4}$} & $\mathrm{Si}(3)-\mathrm{O}(8)$ & $1.579(3)$ \\
\hline & $\mathrm{Si}(3)-\mathrm{O}(10)$ & $1.6167(14)$ \\
\hline & $\mathrm{Si}(3)-\mathrm{O}(11)$ & $1.6261(15)$ \\
\hline & $\mathrm{Si}(3)-\mathrm{O}(9)$ & $1.629(2)$ \\
\hline \multirow[t]{3}{*}{$\mathrm{Cs}_{6} \mathrm{X}(1)$} & $\mathrm{Cs}(3 \mathrm{~A})-\mathrm{X}(1) \times 1.36$ & $3.308(10)$ \\
\hline & $\mathrm{Cs}(3 \mathrm{~B})-\mathrm{X}(1) \times 0.64$ & $3.430(5)$ \\
\hline & $\operatorname{Cs}(4)-X(1) \quad x 4$ & $3.8509(3)$ \\
\hline
\end{tabular}


Table S3. Interatomic distances and bond valence sums for titanium, silicon, and halide (X) polyhedra in $\left[\mathrm{Cs}_{8} \mathrm{Cs}_{8} \mathrm{Cl}_{1.4} \mathrm{~F}_{0.6}\right]\left[(\mathrm{TiO})_{4}\left(\mathrm{Ti}_{6} \mathrm{Si}_{14} \mathrm{O}_{51}\right)\right]$ (2). Bond distances are not listed for the $\mathrm{Si}(3)$ and $\mathrm{Ti}(4)$ polyhedra which reside in the highly disordered portion of the structure.

\begin{tabular}{|c|c|c|c|}
\hline Polyhedron & Interaction & & Distance \\
\hline \multirow[t]{2}{*}{$\mathrm{Ti}(1) \mathrm{O}_{5}$} & $\mathrm{Ti}(1)-\mathrm{O}(8)$ & & $1.75(5)$ \\
\hline & Ti(1)-O(9) & $\mathrm{x} 4$ & $1.854(14)$ \\
\hline \multirow[t]{4}{*}{$\mathrm{Ti}(2) \mathrm{O}_{5}$} & $\mathrm{Ti}(2)-\mathrm{O}(2)$ & & $1.728(9)$ \\
\hline & $\mathrm{Ti}(2)-\mathrm{O}(1 \mathrm{~B})$ & & $1.79(3)$ \\
\hline & $\mathrm{Ti}(2)-\mathrm{O}(3)$ & $\mathrm{x} 2$ & $1.890(12)$ \\
\hline & $\mathrm{Ti}(2)-\mathrm{O}(1 \mathrm{~A})$ & & $1.99(3)$ \\
\hline \multirow[t]{3}{*}{$\mathrm{Ti}(3) \mathrm{O}_{5}$} & $\mathrm{Ti}(3)-\mathrm{O}(10)$ & & $1.64(2)$ \\
\hline & $\mathrm{Ti}(3)-\mathrm{O}(6)$ & $\mathrm{x} 2$ & $1.856(13)$ \\
\hline & $\mathrm{Ti}(3)-\mathrm{O}(11)$ & $\mathrm{x} 2$ & $1.90(3)$ \\
\hline \multirow[t]{3}{*}{$\overline{\mathrm{Si}(1) \mathbf{O}_{4}}$} & $\mathrm{Si}(1)-\mathrm{O}(5)$ & $\mathrm{x} 2$ & $1.598(13)$ \\
\hline & $\mathrm{Si}(1)-\mathrm{O}(9)$ & & $1.634(15)$ \\
\hline & $\mathrm{Si}(1)-\mathrm{O}(7)$ & & $1.668(9)$ \\
\hline \multirow[t]{4}{*}{$\mathrm{Si}(2) \mathbf{O}_{4}$} & $\mathrm{Si}(2)-\mathrm{O}(6)$ & & $1.601(12)$ \\
\hline & $\mathrm{Si}(2)-\mathrm{O}(3)$ & & $1.642(13)$ \\
\hline & $\mathrm{Si}(2)-\mathrm{O}(5)$ & & $1.674(13)$ \\
\hline & $\mathrm{Si}(2)-\mathrm{O}(4)$ & & $1.714(7)$ \\
\hline \multirow[t]{4}{*}{$\mathrm{Cs}_{6} \mathrm{X}(1)$} & Cs(5)-X(1) & & $3.365(10)$ \\
\hline & $\operatorname{Cs}(7)-X(1)$ & $\mathrm{x} 2$ & $3.399(2)$ \\
\hline & $\operatorname{Cs}(6)-X(1)$ & & $3.460(10)$ \\
\hline & $\operatorname{Cs}(6)-X(1)$ & $\mathrm{x} 2$ & $3.6288(16)$ \\
\hline
\end{tabular}


Table S4. Interatomic distances and bond valence sums for titanium, silicon, and chlorine polyhedra in $\left[\mathrm{Rb}_{2} \mathrm{Rb}_{6} \mathrm{Cl}\right]\left[\mathrm{Ti}_{4} \mathrm{Si}_{10} \mathrm{O}_{31} \mathrm{~F}\right]$ (3).

\begin{tabular}{|c|c|c|}
\hline Polyhedron & Interaction & Distance \\
\hline \multirow[t]{3}{*}{$\mathbf{T i}(1) \mathbf{O}_{6}$} & $\mathrm{Ti}(1)-\mathrm{O} / \mathrm{F}(2) \times 2$ & $1.8710(7)$ \\
\hline & $\mathrm{Ti}(1)-\mathrm{O}(3 \mathrm{~A}) \times 2$ & $1.976(4)$ \\
\hline & $\mathrm{Ti}(1)-\mathrm{O}(3 \mathrm{~B}) \times 2$ & $1.994(4)$ \\
\hline \multirow[t]{3}{*}{$\overline{\mathrm{Si}(1) \mathbf{O}_{4}}$} & $\mathrm{Si}(1)-\mathrm{O}(1)$ & $1.598(13)$ \\
\hline & $\mathrm{Si}(1)-\mathrm{O}(9)$ & $1.634(15)$ \\
\hline & $\mathrm{Si}(1)-\mathrm{O}(7)$ & $1.668(9)$ \\
\hline $\mathrm{Si}(2) \mathrm{O}_{4}$ & $\mathrm{Si}(2)-\mathrm{O}(6)$ & $1.609(2)$ \\
\hline \multirow[t]{2}{*}{$\mathrm{Rb}_{6} \mathrm{Cl}(1)$} & $\mathrm{Rb}(2)-\mathrm{Cl}(1) \quad \mathrm{x} 4$ & $3.3249(7)$ \\
\hline & $\mathrm{Rb}(1)-\mathrm{Cl}(1) \quad \mathrm{x} 2$ & $3.3728(7)$ \\
\hline
\end{tabular}

